\title{
Adherencia al tratamiento anti-retroviral para el VIH/SIDA en mujeres: una mirada socio-cultural
}

\author{
Julieta Belmar y Valeria Stuardo
}

\footnotetext{
Universidad de Chile. Facultad de Medicina, Escuela de Salud Pública, Programa de Epidemiología

Los autores declaran no tener conflictos de interés.

Fuentes de financiamiento: Fondo Nacional de Investigación y Desarrollo en Salud FONIS SA1212037: Aspectos

socioculturales implicados en

la adherencia al tratamiento antirretroviral para el $\mathrm{VIH} /$ Sida: desarrollo de indicadores cualitativos para nuevos modelos de intervención.

Recibido: 5 de diciembre de 2016 Aceptado: 11 de julio de 2017

Correspondencia a: Valeria Stuardo Ávila vstuardo@med.uchile.cl
}

\section{Antiretroviral treatment adherence for HIV/AIDS in women: a sociocultural perspective}

Background: Adequate adherence to HAART has a high impact on survival of AIDA patients. There is little consensus on the causes of low adherence to treatment in women, who are in a situation of inequality in terms of prevention and related care. Objectives: To explore and describe the socio-cultural aspects related to the adherence of women to antiretroviral treatment for HIV / AIDS. Material and Methods: Qualitative, exploratory-descriptive study. The study population was focused on Chilean women, who are 18 years of age or older, living with HIV/ AIDS. The sample size was defined by information saturation. In-depth interviews were conducted with 16 women contacted in seven public care centers for people living with HIV (PLHIV) in 4 regions of the country, and it was take into account the saturation of the information. Results: There are several sociocultural factors that determine the level of adherence that women adopt in relation to HAART. The most relevant ones are the vital satisfaction, the imaginary about HIV, the availability of their networks in front of diagnosis and the availability of information are fundamental. Discussion: It is necessary to enter into specific interventions considering the sociocultural aspects and satisfying the psychosocial needs of women. It is imperative that public policies and health teams consider these aspects to improve adherence to HAART.

Key words: HIV, women, adherence, HAART.

Palabras clave: VIH, mujeres, adherencia, terapia anti-retroviral.

\section{Introducción}

$\mathrm{E}$ n Chile, entre 1984 y 2013 se notificaron 31.653 casos de personas con infección por VIH/SIDA y hasta el año 2012 han fallecido 8.298 personas. La tasa de notificación por VIH/SIDA en mujeres muestra un aumento progresivo llegando a 4,7 por 100 mil habts. en el año $2013^{1}$.

El gasto del país en terapia anti-retroviral (TARV) representa $93 \%$ del presupuesto destinado al VIH, y proviene de fondos públicos ${ }^{2}$. Hay más de 22.000 adultos infectados con VIH/SIDA en TARV en el sistema público de salud ${ }^{3}$. Si bien la TARV ha mostrado una elevada eficacia virológica, inmunológica y clínica en el país, la probabilidad de mantenerse con TARV inicial al cabo de tres años es de 57,5\%, lo que encuentra explicación en factores como: toxicidad (47\%), fracaso $(14,7 \%)$, abandono $(18,7 \%)$, y otras $(19,6 \%)^{4}$.

Aunque no existe una definición única de adherencia, hay acuerdo en incorporar en este concepto el compromiso activo y voluntario de las personas en su tratamiento ${ }^{5}$. A nivel internacional entre 20 y $50 \%$ de pacientes bajo TARV presentan una adherencia inadecuada ${ }^{6-8}$, en Chile la proporción de personas que presentan una baja adherencia es cercana a $30 \%{ }^{9}$.
La evidencia muestra que la adherencia a la TARV es vital para demorar o evitar la resistencia a los medicamentos ${ }^{10,11}$. La falta de adherencia se relaciona con mala respuesta al tratamiento, peor reconstitución inmune y mayor riesgo de muerte ${ }^{12}$.

Si bien no existe consenso, se sabe que en la adherencia influyen elementos relacionados con el paciente, la enfermedad, los medicamentos, el equipo asistencial y el sistema de salud ${ }^{5,13}$, destacando aquellos de tipo cognitivo, psicológico y social ${ }^{14-16}$. Diversos estudios plantean la necesidad de explorar y considerar diversas aristas de los contextos de vida de los usuarios de TARV a fin de hacer las adaptaciones pertinentes y apoyar la adherencia ${ }^{17-19}$. La relación sexo-género juega un rol fundamental determinando la forma en que ambos sexos explican y viven el diagnóstico de infección por VIH, así como la posición desde donde lo realizan ${ }^{20}$. En mujeres, la pasividad y subordinación ${ }^{21}$, las ponen en situación de desigualdad en la prevención y cuidados, afectando sus posibilidades de sobrevivencia ${ }^{22}$. Esta posición de subordinación varía en función de otros factores (clase, etnia, edad, etc.) ${ }^{23}$. Otros estudios han mostrado que la adherencia a la TARV en mujeres estaría relacionada con temor a la discriminación ${ }^{24}$, a la divulgación de su diagnóstico ${ }^{25}$, una menor disposición de redes de apoyo ${ }^{15}$, y el cuidado de hijos con infección por $\mathrm{VIH}^{26}$. 
Dentro de las áreas de trabajo prioritarias del marco de acción del tratamiento 2.0 y, como objetivos para el 2020 , se considera relevante mejorar la adherencia a la TARV a través de la optimación de los regímenes médicos y la perspectiva comunitaria, considerando que la desigualdad de género sigue causando un acceso desigual al cuidado $\mathrm{y}$ al tratamiento de poblaciones afectadas ${ }^{27}$.

Así, el propósito del presente trabajo fue explorar y describir los aspectos socio-culturales que están implicados en la adherencia al tratamiento anti-retroviral para el VIH/SIDA en mujeres chilenas.

\section{Materiales y Métodos}

Se realizó un estudio cualitativo, exploratorio-descriptivo. La población de estudio fueron mujeres infectadas con VIH/SIDA de 18 años o más. El tamaño de muestra se definió por saturación de información, que se alcanzó con entrevistas a 16 mujeres. La técnica de recolección de información fue la entrevista en profundidad, aplicada por siete antropólogos capacitados en la patología del VIH.

La pauta de entrevista fue elaborada a partir de la revisión de literatura existente y de los datos aportados por 12 expertos en temas relacionados con la TARV que -a través de un grupo nominal- trabajaron en la definición de las dimensiones mínimas para trabajo de campo. Los convocados participaron de un proceso individual de identificación de elementos conceptuales relevantes, para luego, en una puesta en común, jerarquizar esos elementos en función de las preguntas de investigación. La pauta final se estructuró en tres ámbitos (individual, sociocultural, sistema de salud) y ocho dimensiones (situación persona, historia con el VIH, tratamiento, relación con el medio, estigma y discriminación, asociatividad y redes sociales, expectativas previas de atención, aspectos generales de la atención y los programas). Algunas de las categorías consideradas fueron: imaginario personal del VIH, salud mental, disposición y rol de sus redes sociales frente al diagnóstico, pertenencia a grupos u organizaciones luego del diagnóstico, percepción de estigma y discriminación a propósito de su diagnóstico de infección por VIH, imaginario TARV, experiencia con el uso de TARV, motivos por los que: acepta/no acepta iniciar TARV, etc.

Las mujeres fueron contactadas en siete centros públicos de atención a personas con infección por VIH que concentran más de $60 \%$ de los adultos en TARV en Chile (Hospitales Juan Noé Crevani, Carlos Van Büren, San José, San Borja-Arriarán, El Salvador, Sótero del Río y Hernán Henríquez Aravena) en cuatro regiones del país (Arica y Parinacota, Valparaíso, Metropolitana y Araucanía).

Se consideraron las siguientes posibles categorías de TARV: mujeres en TARV, sin TARV por indicación médica y sin TARV por voluntad propia. Este último perfil de mujeres fue contactado a través de técnica bola de nieve*.

Las entrevistas se aplicaron fuera de los establecimientos. Se realizaron tantas entrevistas como fueron necesarias para bordar la pauta de entrevista y aspectos emergentes que podían aportar a los objetivos del estudio. En todos los casos se utilizó consentimiento informado aprobado por los comités de ética científicos de los servicios de salud involucrados.

Se realizó el análisis narrativo de contenido que corresponde a un análisis cualitativo que rescata la mirada particular de los sujetos en su contexto, considerando las producciones sociales que lo atraviesan. Se utilizaron tanto categorías iniciales como emergentes. La información fue sistematizada usando Atlas ti ${ }^{\circledR}$, que resulta adecuado para el análisis de contenido.

\section{Resultados}

Se entrevistó a 16 mujeres y se analizaron 29 entrevistas (aproximadamente dos entrevistas por mujer). La mediana de edad de las participantes fue de 36 años. Cerca de 50\% estaba en pareja. El 35\% terminó estudios secundarios y $21 \%$ tenía educación universitaria. La principal actividad reportada fue dueña de casa $(35,7 \%)$.

El estudio exploró diversos aspectos relacionados con la adherencia, el resumen se presenta en la Tabla 1. A continuación, el desarrollo de éstos in extenso:

\section{Relación con el VIH}

\section{Imaginario en relación al VIH}

Se atribuye esta patología a grupos específicos como trabajadoras sexuales, consumidores de drogas, homosexuales, personas con múltiples parejas sexuales o gente "sucia". Así mismo, el VIH es asociado a muerte física y social, expresada en una apariencia demacrada, muerte prematura, discriminación laboral, social, afectiva y en el término de los proyectos de vida, debido a una muerte anunciada.

\section{Percepción de riesgo en relación al VIH previo al diagnóstico}

Baja o nula, debido a que no se consideran pertenecientes a ningún grupo de "riesgo" ni haberse vinculado con personas pertenecientes a ellos.

\footnotetext{
* Nota del Editor: Los investigadores utilizan este método de muestreo si la muestra para el estudio es muy rara o si está limitada a un subgrupo muy pequeño de la población. Este tipo de técnica de muestreo funciona en cadena. Luego de observar al primer sujeto, el investigador le pide ayuda a él para identificar a otras personas que tengan un rasgo de interés similar.
} 
Tabla 1. Resumen de aspectos asociados a la adherencia a TARV en mujeres con infección por VIH

\begin{tabular}{|c|c|c|}
\hline Dimensión & Aspectos & Descripción \\
\hline \multirow[t]{5}{*}{ Relación con el VIH } & Imaginario en relación al VIH & $\begin{array}{l}\text { VIH asociado a grupos específicos cuyo comportamiento es sancionado socialmente. } \\
\text { También a muerte física y social }\end{array}$ \\
\hline & $\begin{array}{l}\text { Percepción de riesgo en relación al VIH previo } \\
\text { al diagnóstico }\end{array}$ & Baja o nula, debido a que no se consideran pertenecientes a ningún grupo de "riesgo" \\
\hline & Cambios en su vida luego del diagnóstico & Afecta fundamentalmente a su situación de pareja y su vida social \\
\hline & $\begin{array}{l}\text { Centros de atención de personas con infección } \\
\text { por VIH }\end{array}$ & $\begin{array}{l}\text { Percepción de buen trato, acogida y privacidad. La información recibida se valora como } \\
\text { insuficiente }\end{array}$ \\
\hline & Salud mental & $\begin{array}{l}\text { Fue habitual encontrar trastornos depresivos o ansiosos y la consulta con especialistas. } \\
\text { Sin embargo, estas instancias se evalúan negativamente }\end{array}$ \\
\hline \multirow[t]{3}{*}{$\begin{array}{l}\text { Relación con otros y } \\
\text { satisfacción vital }\end{array}$} & Redes sociales & $\begin{array}{l}\text { En general, se reportan redes escasas, compuestas por familia o amigos. Quienes po- } \\
\text { seen red más amplia, reportan sentir mayor apoyo afectivo }\end{array}$ \\
\hline & Estigma y discriminación & $\begin{array}{l}\text { Se relata discriminación en centros de salud no especializados en VIH, al estar hospita- } \\
\text { lizadas, conocer nuevas parejas y por sus familias al inicio del diagnóstico. Se observa } \\
\text { discriminación internalizada y baja lucha por la defensa de sus derechos }\end{array}$ \\
\hline & Satisfacción y expectativas en relación a la vida & $\begin{array}{l}\text { Se observa tristeza, desesperanza y bajo nivel de satisfacción. La mayoría considera que } \\
\text { sus sueños se truncaron por el VIH y que su vida no tiene sentido salvo para cuidar a } \\
\text { sus hijos. También se reporta vida solitaria y estresante por responsabilidad económica } \\
\text { y parental }\end{array}$ \\
\hline
\end{tabular}

Relación con la terapia Conocimiento y/o imaginario acerca de la TARV anti-retroviral (TARV) previo al diagnóstico

Motivos para iniciar o abandonar la TARV
En general se maneja muy poca información y principalmente es de carácter positivo. Como negativo se mencionan efectos secundarios

Iniciar por primera vez: estar bien para sus hijos y porque es bueno para su salud Rechazar la primera vez: temor a efectos secundarios y considerarla innecesaria en ese momento

Iniciar TARV después de haberla rechazado: enfermedad grave y/o persuasión del equipo médico

Abandono de tratamiento: Desesperanza, desagrado por tomar medicamentos y efectos secundarios

Retomar TARV: enfermedad grave, molestias físicas o persuasión relacionada con su compromiso con los hijos

\begin{abstract}
"No porque con los síntomas, era como alguien... yo no soy paciente, yo no soy de esas... o si, se me paso por la cabeza, a eso le pasan a las que trabajan en la esquina porque ya andan con uno y otro, pero nunca pensé... nunca se me pasó por la mente que yo..." (A007, No TARV VP).
\end{abstract}

\section{Cambios en su vida luego del diagnóstico}

Mujeres que tenían pareja al ser diagnosticadas, decidieron seguir con ellas a pesar de problemas surgidos por el propio diagnóstico o relacionados con consumo de alcohol y/o drogas y violencia física y/o sexual.

"Con lo que tengo estoy jodida pal resto de mi vida, y a lo mejor que esa es la rabia, porque un día me quisiera apartar de él, pero no me gusta la soledad, nadie me va a querer con lo que tengo..." (A009, TARV).
Por su parte, quienes no tenían pareja en ese momento, manifiestan que la búsqueda de un compañero ha sido difícil y dolorosa por el temor al rechazo o la discriminación efectiva. Se lamentan la limitación para formar y para tener amistades y vida social, por temor al rechazo o lástima.

Centros de atención de personas con infección por VIH

Se reporta sensación de escucha, acogida, apoyo y confianza. En algunos casos, se vislumbra un actuar coercitivo. La información sobre la enfermedad y tratamiento recibida, por parte del equipo médico, se evalúa como insuficiente. Se valora la confidencialidad, aunque se sugiere mejorar privacidad en salas de espera y hospitalización.

\section{Salud mental}

Fue habitual la presencia de trastornos depresivos o ansiosos y la consulta con especialistas del centro 
asistencial o de servicios privados. En general - aunque especialmente en mujeres no adherentes - existe una evaluación negativa de estas instancias, argumentando incomodidad antes los temas que deben tratar o desacuerdo con la toma de medicamentos debido sus efectos secundarios. Se reportan intentos de suicidio y consumo problemático de alcohol luego del diagnóstico.

\section{Relación con otros y satisfacción vital}

\section{Redes sociales}

En general se reportan redes escasas, compuestas por familia de origen y algunos amigos o exclusivamente por pareja e hijos. En este grupo de mujeres hay quienes han vivido su diagnóstico en soledad, quienes lo han revelado a pareja e hijos y cuentan con su apoyo y quienes han recibido una aceptación mediada por la discriminación. Las mujeres con red de apoyo amplia han comunicado a mayor número de personas su diagnóstico y relatan mayor apoyo afectivo.

\section{Estigma y discriminación}

Se relata discriminación en centros de salud no especializados en la patología del VIH, al estar hospitalizadas, conocer nuevas parejas y por sus familias al inicio del diagnóstico. La mayoría de las mujeres reporta haber escuchado burlas y dichos discriminatorios en relación a las personas infectadas con VIH.

Respecto a la auto discriminación, se destaca el aceptar ser segregadas por sus familias al inicio del diagnóstico, pensar que ningún hombre las pudiera querer por tener la infección por VIH y sentir que pudieron ser responsables por haber sido demasiado independientes o por tener varias parejas sexuales.

Respecto del conocimiento y/o lucha por el cumplimiento de derechos y leyes que las protejan contra la discriminación y el maltrato, se observó que no es un tema prioritario para las entrevistadas, pero que tiene algunos aspectos que son más conocidos como el derecho a la confidencialidad del diagnóstico y que legalmente no pueden ser rechazadas en un trabajo por su condición. Sin embargo, frente a algunos atropellos, las entrevistadas no manejan las herramientas para el ejercicio de sus derechos.

\section{Satisfacción y expectativas en relación a la vida}

Se observa tristeza, desesperanza y bajo nivel de satisfacción. La mayoría considera que sus sueños se truncaron por esta enfermedad y que su vida no tiene sentido salvo para cuidar a sus hijos. También se reporta una vida solitaria y estresante producto de la responsabilidad económica y parental que recae sobre ellas. Específicamente, quienes han sido irregulares en su tratamiento, declaran no tener deseos de vivir y sensación de infelicidad permanente.

\section{Relación con la terapia anti-retroviral}

Conocimiento y/o imaginario acerca de la TARV previo al diagnóstico

En general se maneja muy poca información y principalmente es de carácter positivo. Son mencionados beneficios terapéuticos de TARV y terapia personalizada. Como aspecto negativo se mencionan los efectos secundarios.

\section{Motivos para iniciar o abandonar la TARV}

Para iniciarla por primera vez se señala estar bien para sus hijos y la convicción de que el inicio temprano las favorece. Los motivos para no aceptar TARV la primera vez que les fue indicada fueron temor a efectos secundarios y creer que aún no la necesitaban. Los motivos para iniciar TARV después de haberla rechazado fueron enfermedad grave y/o persuasión del equipo médico. Dentro de los motivos para abandonar el tratamiento se mencionan sensación de soledad y desesperanza que las lleva a ver el abandono de la terapia como una forma de terminar rápido con su vida.

\begin{abstract}
"Yo creo que a lo mejor ya, ya estoy con la idea de que en realidad no es que, a lo mejor, no sé si estaré sola o no, pero yo me siento demasiado sola, entonces de repente digo "¿Y a quién le hago falta?" yo no me voy a suicidar, pero a lo mejor sin tomarme los medicamentos me llegará mi tiempo más rápido y me voy poh'..." (E007, No TARV VP).
\end{abstract}

También desagrado por tomar medicamentos y efectos secundarios de la TARV. Finalmente, dentro de los motivos para retomar TARV se reportan enfermedad grave, molestias físicas o persuasión relacionada con su compromiso con los hijos. Dentro de los motivos para adherir completamente destacan auto-responsabilidad, mantenerse sanas para criar a sus hijos y la necesidad de mantener a la familia.

\section{Discusión}

El estudio mostró que la adherencia en mujeres es un proceso que resulta de la interacción de diversos elementos, que han sido esquematizados en la Figura 1. Algunos de estos factores, han sido mencionados en estudios que analizan el fenómeno a nivel genera ${ }^{5,14,28}$. Sin embargo, éstos no consideran variables transversales como el género, etnia o clase social entre otros, que permiten explicar las expresiones particulares que cada factor adopta en los distintos grupos ${ }^{29}$, particularmente el imaginario social sobre la infección por VIH, entendido como las representaciones colectivas que los seres humanos crean 
Figura 1. Esquema de factores implicados en la adherencia a TARV en mujeres.

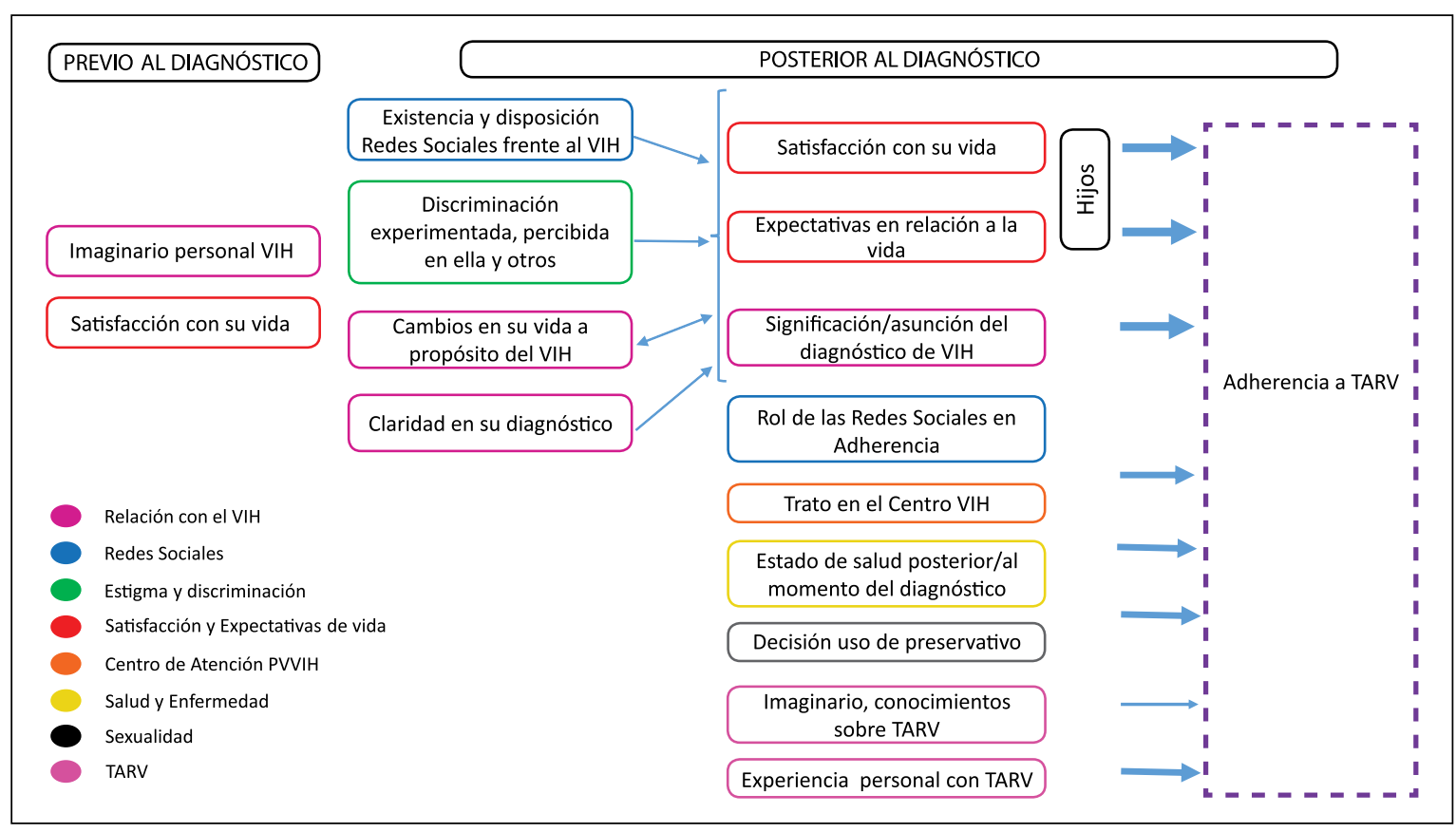

y desarrollan para dar sentido a sus experiencias, en función de la realidad vivida ${ }^{30}$. De acuerdo a los resultados del presente estudio, la experiencia de género resulta central para comprender cómo las mujeres significan y enfrentan el diagnóstico de infección por VIH y, si bien esta experiencia es vivenciada de diferentes maneras en función del peso de las demás relaciones sociales ${ }^{23}$, resulta una variable determinante.

Para Stolcke ${ }^{31}$, las diferencias en la clase, el género y la etnia u otras categorías de análisis que afectan la salud de las personas, no significan nada en sí mismas, sino que adquieren el valor o significado simbólico que cada sociedad o cultura le asigne y legitime. Además, en el caso de las mujeres, el género se suma a las otras desigualdades que también vivencian los hombres, produciéndose una doble desventaja desde el inicio de sus vidas.

En la relación específica con la adherencia a TARV en mujeres, un primer elemento que impacta y que ha sido mencionado en otras investigaciones, es el estigma que aún rodea a la infección por $\mathrm{VIH}^{24,32}$, el que actúa sobre el imaginario personal de las mujeres en relación al VIH, la discriminación vivida y percibida, y la propia discriminación hacia el imaginario de perfil de personas afectadas por el VIH. Como muestran los resultados, el estigma está asociado no sólo a padecer de esta infección, sino a ser una mujer que vive con ella, lo que es motivo de doble discriminación.

Un segundo elemento, son los cambios que han debido realizar o creen que deben realizar a propósito del diagnóstico de infección por VIH, los que también están mediados por el género, pues por ejemplo, tal como se hombre el encontrar una nueva pareja en esa condición, tener hijos o ser considerados "promiscuos". Así, tanto el estigma como la discriminación y los cambios implementados terminan por afectar o determinar el nivel de satisfacción vital, expectativas de vida y el significado que atribuyen a la enfermedad.

Según los presentes resultados y tal como se ha visto en otros estudios de adherencia a TARV ${ }^{5,33,34}$, estos últimos elementos son poderosos determinantes del proceso de adherencia, pudiendo ser influenciados o modificados por el apoyo recibido de las redes cercanas en aspectos específicos del VIH, el trato percibido en el centro de salud, el estado de salud física y mental experimentado luego del diagnóstico y los conocimientos, experiencia e imaginario de uso de TARV.

Finalmente, al igual que lo señalan otras investigaciones $^{5,28,33}$, hemos visto que la biografía de cada una de las mujeres, las vulnerabilidades y la satisfacción previa al diagnóstico, afectan de manera específica el camino de la adherencia. Gran parte de estos factores deben ser explicados a la luz de un análisis de género que permita comprender el carácter estructural, aunque modificable, de las vivencias y significados atribuidos al fenómeno de la adherencia $^{22,35}$.

Un artículo reciente expone sobre cómo las diferencias de género influyen en la adherencia, evidenciando que las intervenciones futuras tendrán que ir más allá del modelo observó en otro estudio ${ }^{29}$, no es preocupación para un 
de tratamiento directamente observado, siendo adaptadas a subgrupos específicos y considerando aspectos socioculturales y el apoyo sociall'.

En general, la notificación de tener la infección por VIH fue acompañada de sintomatología depresiva, lo que se ha asociado a menor adherencia ${ }^{37}$. Esto se relaciona con un imaginario de la infección por VIH asociado a la muerte y habla del fracaso del sistema de salud para erradicar esta idea.

Así, los resultados de este estudio permiten reforzar tres puntos en relación con la adherencia a la TARV en mujeres:

- que la satisfacción con la vida previa al diagnóstico, el imaginario que se maneja en relación al VIH, la discriminación internalizada y la disposición de las redes frente al diagnóstico son elementos fundamentales en la adherencia ${ }^{32,38}$;

- que dichos elementos pueden estar atravesados por la condición de género de las entrevistadas que define -o al menos refuerza- contextos de vulnerabilidad específicos desde la prevención hasta el tratamiento ${ }^{22,39}$ $\mathrm{y}$;

- que hay variables susceptibles de ser modificadas y abordadas en el contexto de la prevención, diagnóstico $\mathrm{y}$ tratamiento.

El impacto positivo de la TARV no ha sido acompañado de mayor aceptación social de las mujeres infectadas con VIH, esto afecta su adherencia. Asimismo, su bajo empoderamiento respecto de las normativas legales y DD.HH. conduce a la transgresión de los mismos. Es necesario que los equipos de salud y las políticas públicas asociadas, consideren los aspectos socioculturales y contextos de vulnerabilidad en las estrategias destinadas a mejorar la adherencia a la TARV de las mujeres.

Agradecimientos. A los/las Drs. Beatriz Marincovich, Marcelo Wolff, Carlos Gallo, Elizabeth Barthel, Martin
Lasso, Esteban Cortez, Carolina Chahín y Rossana Benítez y a los equipos locales de los centros de asistenciales para pacientes con infección por VIH por el interés demostrado al participar en este proyecto. A todas las entrevistadas por su generosa contribución, por sus historias de vida y por la confianza depositada.

\section{Resumen}

Introducción: La adecuada adherencia a la TARV tiene un alto impacto en la sobrevida en el SIDA. Existe poco consenso sobre las causas de la baja adherencia al tratamiento en mujeres, estando éstas en una situación de desigualdad en términos de prevención y cuidados relacionados. Objetivos: Explorar y describir los aspectos socioculturales vinculados a la adherencia de mujeres al tratamiento anti-retroviral para el VIH/SIDA. Material y Métodos: Estudio cualitativo, exploratoriodescriptivo. La población de estudio fueron mujeres chilenas de 18 de edad o más con infección por VIH/ SIDA. El tamaño de la muestra se definió por saturación de información. Se realizaron entrevistas en profundidad a 16 mujeres contactadas en siete centros públicos de atención a personas con infección por VIH en cuatro regiones del país, atendiendo al criterio de saturación de la información. Resultados: Existen diversos factores socio-culturales que determinan el grado de adherencia que las mujeres adoptan en relación al TARV. Los más relevantes son la satisfacción vital, el imaginario sobre el VIH, la disposición de sus redes frente al diagnóstico y la disponibilidad de información. Discusión: Se hace necesario incursionar en intervenciones específicas considerando los aspectos socio-culturales y satisfaciendo las necesidades psicosociales de las mujeres. Se hace imprescindible que las políticas públicas y los equipos de salud consideren dichos aspectos para mejorar la adherencia a la TARV.

\section{Referencias bibliográficas}

1.- Ministerio de Salud de Chile (MINSAL) GDC. Informe Evolución del VIH/Sida 1984-2013. Chile. 2015. http://www.scielo.cl/pdf/rci/v32s1/ art03.pdf

2.- Organización Panamericana de la Salud (OPS). Tratamiento antirretroviral bajo la lupa: un análisis de salud pública en Latinoamérica y el Caribe. Washington. D.C; 2012. .paho.org/hq/ index.php?option $=$ com_docman\&task $=$ doc_vie w\&gid $=23711 \&$ itemid\&Itemid $=270$

3.- Ministerio de Salud de Chile (MINSAL). Informe Nacional de Progreso sobre SIDA en Chhile, GARPR 2014.
4.- MINSAL-CONASIDA. Evaluación de impacto del programa de acceso expandido a tratamiento antirretroviral Cohorte chilenade SIDA. 2007. http://web.minsal.cl/portal/url/item/853814 14c60311a9e04001011e015920.pdf

5.- Organización Panamericana de la Salud (OPS). Experiencias exitosas en el manejo de la adherencia al tratamiento antirretroviral en Latinoamérica. Washington. D.C; 2010.

6.- Muñoz-Moreno J A, Fumaz C R, Ferrer M $\mathrm{J}$, Tuldrà $\mathrm{A}$, Rovira $\mathrm{T}$, Viladrich $\mathrm{C}$, et al; TS validation team. Assessing self-reported adherence to HIV therapy by questionnaire:
The SERAD (self-reported adherence) study. AIDS Res Hum Retroviruses 2007; 23 (10): 1166-75.

7.- Wiener L, Riekert K, Ryder C, Wood LV. Assessing medication adherence in adolescents with HIV when electronic monitoring is not feasible. AIDS Patient Care STDS 2004; 18 (9): 527-38.

8.- Nieuwkerk P T O F. Self-reported adherence to antiretroviral therapy for HIV-1 infection and virologic treatment response: a metaanalysis. J Acquir Immune Defic Syndr 2005; 38: $445-8$

9.- Ministerio de Salud de Chile (MINSAL) GDC. Calidad de vida en personas que viven 
con VIH/SIDA. Informe final de resultados. 2007.

10.- Organización Panamericana de la Salud (OPS). Tratamiento antirretroviral de la infección por el VIH en adultos y adolescentes en Latinoamérica y el Caribe: en la ruta hacia el acceso universal. 2008. http://apps.who.int/ medicinedocs/es/m/abstract/Js19814es/

11.- PNUD. Estudio de la medición de la adherencia a la terapia antirretroviral (TAR) de personas con VIH de la red de hospitales del Ministerio de Salud de El Salvador. 2013.

12.- García de Olalla P, Knobel H, Carmona A, Guelar A, López-Colomes J L C J. Impact of adherence and highly active antiretroviral therapy on survival in HIV-infected patients. J Acquir Immune Defic Syndr 2002; 30 (1): 105-10.

13.- Arrivillaga M, Ross M, Useche B, Springer A, Correa D. Applying an expanded social determinant approach to the concept of adherence to treatment: the case of Colombian women living with HIV/AIDS. Women's Health Issues 2011; 21 (2): 177-83.

14.- Oscar A, Coll LD, Chumbimune L, Díaz C, Judith D, Reyes M. Factores asociados a la no adherencia al tratamiento antirretroviral de gran actividad en adultos infectados con el VIH-sida. An Fac Med UNMSM 2009; 70 (4): 266-72.

15.- Knowlton A R, Yang C, Bohnert A, Wissow L, Chander G A J. Informal care and reciprocity of support are associated with HAART adherence among men in Baltimore, MD, USA. AIDS Behav 2008; 144 (5): 724-32.

16.- Boateng D, Kwapong G D, Agyei-Baffour P. Knowledge, perception about antiretroviral therapy (ART) and prevention of mother-tochild-transmission (PMTCT) and adherence to ART among HIV positive women in the Ashanti Region, Ghana: a cross-sectional study. BMC Womens Health 2013; 13 (1): 2-8.

17.- Stern E, Colvin C, Gxabagxaba N, Schutz Ch, Burton R and M G. Conceptions of agency and constraint for HIV-positive patients and healthcare workers to support long-term engagement with antiretroviral therapy care in Khayelitsha, South Africa. African J AIDS Res 2017; 16 (1).
18.- Barroso J, Leblanc N M, Flores D. It's not just the pills: A qualitative meta-synthesis of HIV antiretroviral adherence research. Assoc Nurses AIDS Care 2017.

19.- Inzaule S C, Hamers R L, Kityo C, Rinke de Wit T F, Roura M, Adefolalu A, et al. Longterm antiretroviral treatment adherence in HIV-infected adolescents and adults in Uganda: A qualitative study. PLoS One 2016; 11 (11): e0167492.

20.- Herrera C, Campero L. La vulnerabilidad e invisibilidad de las mujeres ante el VIH / SIDA constantes y cambios en el tema. Salud Publica Mex 2008; 44 (655): 554-62.

21.- Tallis V. Género y VIH/SIDA. Informe General. 2005.

22.- García Martínez A. Educación y prevención del SIDA. An Psicol 2005; 21: 50-7.

23.- Ministerio de la Protección Social F de P de las NU y U de C. Factores de Vulnerabilidad a la infección por VIH en mujeres. Colombia; 2009.

24.- Mhode M. Lived Experiences of stigma and discrimination among people on antiretroviral therapy: a qualitative study in Ilala Municipality. Dar Es Salaam 2013; 2016.

25.- Arrivillaga-Quintero M. Análisis de las barreras para la adherencia terapéutica en mujeres colombianas con VIH/SIDA: cuestión de derechos de salud. Salud Publica Mex 2010; 52 (118): 350-6.

26.- Arrivillaga M, Ross M, Useche B, Alzate M L, Correa D. Social position, gender role, and treatment adherence among Colombian women living with HIV/AIDS: social determinants of health approach. Rev Panam Salud Publica 2009; 26 (6): 502-10.

27.- Organización Panamericana de la Salud (OPS). Marco de acción del tratamiento 2.0: impulsionando la próxima generación del tratamiento, la atención y el apoyo. 2011; 30.

28.- Margulies S, Barber N, Recoder M. VIH-SIDA y adherencia al tratamiento. Enfoques y perspectivas. Antipoda 2006; 3 (3): 281-300.

29.- Stuardo Ávila V, Manríquez Urbina J M, Fajreldin Chuaqui V, Belmar Prieto J, Valenzuela Santibáñez V. Model of socio- cultural dimensions involved in adherence to antiretroviral therapy for HIV/AIDS in public health care centers in Chile. AIDS Care Psychol Socio-Medical Asp AIDS/HIV. 2016; 28 (11).

30.- Pintos J L. Los imaginarios sociales: la nueva construcción de la realidad social. Terrae, editor. 1995.

31.- Stolcke V. Antropología del género. Ensayos de antropología cultural. Homenaje a Claudio Esteva-Fabregat. Editorial Ariel, S.A., Barcelona, 1996; pp. 335-44.

32.- Tarimo E A M, George J. Providing antiretroviral therapy in the context of selfperceived stigma: A mixed methods study from Tanzania. Tanzan J Health Res 2014; 16 (2): $1-12$.

33.- Muñoz Sánchez A I, Cruz Martínez Ó A, Rubiano Mesa Y L. Trabajadores de la salud y sus significados en torno a la adherencia al tratamiento de la tuberculosis. Enfermería Glob 2013; 31: 86-108.

34.- Estrada J H, Martínez A G, Abadía C E. Factores biosociales y percepciones de una vida con calidad: reto para la adherencia antirretroviral en pacientes con VIH. Rev Fac Nac Salud Pública 2008; 26 (2): 134-42.

35.- García-Sánchez Inés. Diferencias de género en el VIH/SIDA. Gac Sanit 2004; 18 (5).

36.- Puskas C, Hogg R. Unlocking adherence: is gender the key? Lancet HIV 2014; 2 (1)

37.- Varela M, Galdames S. Depresión y adhesión a terapia anti-retroviral en pacientes con infección por VIH atendidos en el Hospital San Pablo de Coquimbo, Chile. Rev Chilena Infectol 2014; 31 (3): 323-8.

38.- Wagner G J, Bogart L M, Galvan F H, Banks D K D. Discrimination as a key mediator of the relationship between posttraumatic stress and HIV treatment adherence among African American men. J Behav Med 2012; 35 (1): 8-18.

39.- Palma I, Abarca H, Moreno C. Estrategias de prevención en salud sexual y reproductiva en jóvenes de América Latina y el Caribe. Hacia una nueva síntesis de enfoques. 2002. 